\title{
PRICING OPTIONS IN A DELAYED MARKET DRIVEN BY LE'VY NOISE
}

\author{
ISMAIL HAMED ELSANOUSI* \\ Department of Mathematics, Faculty of Sciences Al-Baha University,P.O.Box-1988, Alaqiq, \\ Al-Baha-65431, Saudi Arabia \\ *Corresponding author: i_elsanousi@hotmail.com
}

\begin{abstract}
In this paper we studied stochastic delayed differential equations driven by Le'vy noise. The analogue of Itô formula is considered. The Black-Scholes formula analogue for Vanilla call option price formula is derived.
\end{abstract}

\section{INTRODUCTION}

In this paper we studied the Stochastic Delay Differential Equations driven by Le'vy noise which arise in many applications of stochastic analysis in finance specifically in pricing of options security markets. As known such systems are quite hard to study due to their lack of Markovianity which is a key property for the study of option prices. Basically, the difficulties arises from the fact that delay systems have, in general, an infinite dimensional nature.

The model for the stock price $\zeta(t)$ that we consider satisfies a stochastic delay differential equation driven by Le'vy noise with volatility $\sigma$ depending on time $t$ and the path $\zeta_{t}=\{\zeta(t+\theta), \theta \in[-\tau, 0]\}$ called a level and past-dependent volatility. An analogue of Itô's formula for such a stochastic systems is obtained.

Received March 29 $9^{\text {th }}, 2021$; accepted April 23 ${ }^{\text {rd }}, 2021$; published May $11^{\text {th }}, 2021$.

2010 Mathematics Subject Classification. 60G40, 34K50.

Key words and phrases. stochastic delay equations; Black-Scholes formula; (B,S)-securities market.

(C)2021 Authors retain the copyrights of their papers, and all open access articles are distributed under the terms of the Creative Commons Attribution License. 
An option price value of the form

$$
G\left(t, \zeta_{t}\right)=\int_{-\tau}^{0} e^{r \theta} F(\zeta(t+\theta), \zeta(t), t) d \theta
$$

is studied when $F$ is in $\mathbb{C}^{0,1,2}\left(\mathbb{R} \times \mathbb{R} \times \mathbb{R}^{3}\right)$. A special case of $G\left(t, \zeta_{t}\right)$ of the form

$$
G\left(t, \zeta_{t}\right)=g_{1}(\zeta(t), t)+\int_{-\tau}^{0} e^{r \theta} g_{2}(\zeta(t+\theta), t) d \theta
$$

when $g_{1}(\zeta(t), t)$ is a classical Black-Scholes call option is studied. The partial differential equation of BlackScholes type is derived for such option.

\section{Notations and Preliminaries}

Let us consider a probability space $(\Omega, \digamma, \rho)$ on which is defined $\left((B(t))_{t \geq 0},(\eta(t))_{t \geq 0}\right)$ where $\left((B(t))_{t \geq 0}\right.$ and $\left.(\eta(t))_{t \geq 0}\right)$ are independent stochastic processes

$-\left((B(t))_{t \geq 0}\right.$ is a standard Brownian motion with respect to its natural filtration, $\left.-(\eta(t))_{t \geq 0}\right)$ is a pure jump Le'vy process.

The Poisson random measure $N(t)$ of the process $\eta$ is defined by

$$
N(t, A)=\sum_{\zeta \in[0, t]} I_{A}\left(\eta(s)-\eta\left(s^{-}\right)\right), \quad A \subset \mathbb{R} .
$$

The Le'vy measure $\nu$ of the process $\eta$ is supposed to be $\int_{\mathbb{R}} z^{2} \nu(d z)<+\infty$, since $\left.(\eta(t))_{t \geq 0}\right)$ is a pure jump $\nu(\{0\})=0$.

Define the measure-valued process $(\tilde{N}(t))_{t \geq 0}$ by

$$
\tilde{N}(t, d z):=N(t, d z)-\nu(d z)
$$

so that the compensated poisson random measure is

$$
\tilde{N}(d t, d z):=N(d t, d z)-\nu(d z) d t
$$

Let $\left(\digamma_{t}\right)_{t \geq 0}$ be the filtration generated by the process $B(t)$ and $\eta(t)$ as defined above. Since $B$ and $\eta$ are assumed independent, then $B($.$) and \tilde{N}(., A)$ are still Brownian motion and square integrable martingale with respect to the filtration $\left(\digamma_{t}\right)_{t \geq 0}$. 


\subsection{Stochastic Delay Differential Equations Driven By Le'vy Noise}

Consider the following Sdde:

$$
\begin{aligned}
d x(t)= & \mu\left(t, x_{t}\right) d t+\sigma\left(t, x_{t}\right) d w(t)+\int_{\mathbb{R}} \gamma\left(x_{t}^{-}, z\right) \tilde{N}(d t, d z), t \in[0, T] \\
& x_{0}=x(\theta), \quad \theta \in[-r, 0]
\end{aligned}
$$

where $\mu:[0, T] \times D \rightarrow \mathbb{R}^{n}, \sigma:[0, T] \times D \rightarrow \mathbb{R}^{n d}, \gamma: D \times D \rightarrow \mathbb{R}$ are predictable processes.

For the unknown process $(x(t))_{t \in[-r, T]}$ in $\mathbb{R}, T<\infty$ is a fixed finite time, $x(t)$ is the value of $x$ at $t \in[0, T]$ and $x_{t}$ its segment, i.e. its value in the past time interval $[t-r, t]$ i.e. $x_{t}():.[-r, 0] \rightarrow \mathbb{R}$ defined by $x_{t}(\theta): x(t+\theta)$ for all $\theta \in[-r, 0]$.

The initial data $x(\theta)$ is assumed to be in the space $D:=D([-r, 0], \mathbb{R})$ of Càdlàg random variables from $[-r, 0]$ to $\mathbb{R}$.

Hypotheses 2.1 (i). There exists constant $L>0$ such that for all $t \in[0, T]$ and for all $x_{1}, x_{2} \in D$,

$$
\left|\mu\left(t, x_{1}\right)-\mu\left(t, x_{2}\right)\right|+\left|\sigma\left(t, x_{1}\right)-\sigma\left(t, x_{2}\right)+\int_{\mathbb{R}}\right| \gamma\left(x_{1}, z\right)-\gamma\left(x_{2}, z\right)|\nu(d z) \leq L| x_{1}-x_{2} \mid .
$$

(ii). The functions $\mu, \sigma, \gamma$ satisfy the linear growth condition, i.e. there exists a constant $K>0$ such that for all $x \in D$,

$$
|\mu(t, x)|+|\sigma(t, x)|+\int_{\mathbb{R}}|\gamma(x, z)| \nu(d z) \leq K(1+|x|) .
$$

Theorem 2.1. Suppose that hypotheses (i) and (ii) hold. Then there exists a unique $C a^{\prime} d l a^{\prime} g$ adapted solution to equation (2.1). For proof we refer to [7].

\section{The infinitesimal operator of the solution of equation (2.1)}

In reformulation of equation (2.1) in infinite dimension, the following linear stochastic evolution equation in the space $H$

$$
d x(t)=A x(t) d t+<\sigma, x(t)>-\hat{n} d w(t)+\int_{\mathbb{R}}<r(z), x\left(t^{-}\right)>-\hat{n} \tilde{N}(d t, d z)
$$

will represents equation (2.1) in the sense that

$$
x(t)=\left(x_{0}(t), x_{1}(t)\right)=(x(t), x(t+\theta): \theta \in[-T, 0], \forall t \geq 0
$$


where $\mathrm{A}$ is defined on

$$
D\left(A=\left\{y=\left(y_{0}, y_{1}(.)\right) \in H ; y_{1}(.) \in W^{1,2}([-1,0], \mathbb{R}), y_{0}=y_{1}(0)\right\}\right.
$$

by $A y=\left(\mu_{0} y_{0}+\int_{-T}^{0} \mu_{1}(\theta) y_{1}(\theta) d \theta+\mu_{2} y_{1}(-T), y_{1}^{\prime}().\right)$ is the generator of a strongly continuous semigroup $(s(t))_{t \geq 0}$ on $H$. Here $\hat{n}=(1,0) \in H$ and $x\left(t^{-}\right):=\lim _{s \uparrow t} x(s)=\lim _{s \uparrow t}(x(s), s(s+)$.$) with the limit taken in H$.

Theorem 2.1. Let $y \in C$ and let $x(. ; y), x(. ; y)$ representing the solutions of $(2.1)$ and $(2.1)^{\prime}$ respectively. Then $x(. ; y)$ represents $s(. ; y)$ in the sense that

$$
x(t ; y)=(x(t ; y), x(t+\theta ; y): \theta \in[-T, 0]), \forall t \geq 0 .
$$

For proof see [11].

The infinitesimal operator of the process $x(., 0)$ is formally defined as

$$
\begin{array}{r}
{[L \phi](y):=<A y, \varphi_{y}(y)>+\frac{1}{2}<\sigma, y>\varphi_{y_{0} y_{0}}(y)+} \\
\int_{\mathbb{R}}\left[\varphi(y+<r(z), y>\hat{n})-\varphi(y)-\varphi_{y_{0}}(y)<r(z), y>\right] \nu(d z), \quad y \in D(A), \varphi \in C^{2}(H ; \mathbb{R}) .
\end{array}
$$

\section{Option Price Formula}

Assume that the stock price satisfy the following stochastic delay differential equation of the form

$$
\begin{gathered}
d s(t)=r s(t) d t+\sigma\left(t, s_{t}\right) d w(t)+\int_{\mathbb{R}} \gamma\left(s_{t}^{-}, z\right) \tilde{N}(d t, d z), t \in[0, T] \\
s(0)=y_{0}, s(\theta)=y_{1}(\theta), \quad \theta \in[-r, 0]
\end{gathered}
$$

where $y:=\left(y_{0}, y_{1}().\right) \in C$ is positive. Here $C$ is the subspace of the Hilbert space $H:=\mathbb{R} \times L_{-r}^{2}:=$ $\mathbb{R} \times L^{2}([-r, 0], \mathbb{R})$ whose inner product $<., .>$ is

$$
<., .>=<., .>_{\mathbb{R}}+<., .,>_{L_{r}^{2}}
$$

$C:=y \in H: y_{1}($.$) admits a C a \grave{d l} a^{\prime} g$ representative.

In (3.1) $r \in \mathbb{R}, \sigma:=\left(\sigma_{0}, \sigma_{1}(.) \in H, \gamma():.=\left(\gamma_{0}, \gamma_{1}().().\right) \in L^{2}(\mathbb{R}, \nu ; H)\right.$ are functional parameters.

\section{Analogue of Black-Scholes formula for Vanilla call option price:}

Suppose that the financial market under consideration as follows: (i). A risk free asset given by

$$
d s_{0}(t)=r\left(s_{0}\right)(t) d t ; t \in[0 . T]
$$

(ii). A risky asset given by equation (3.1)-(3.2). 
A portfolio in such market is an $F_{t}$ predictable process $\Pi(t)$ representing the number of units held at time $t$ of the assets number $0,1, \ldots, n$ respectively, then the wealth process $x(t)=x^{\Pi}(t)$ associated to the portfolio $\Pi$ is defined to be:

$$
x^{\Pi}(t)=\Pi(t) s(t)=\sum_{i=1}^{n} \Pi_{i}(t) s_{i}(t) .
$$

\section{Absence of arbitrage}

In order to have no arbitrage opportunities in the considered market, the return from the portfolio must be risk-free with interest rate $r$.

In what follows we adopt that $\Pi(t)$ is riskless during $[t, t+d t]$ and instantaneously earn the same rate of return as other short-term risk-free assets.

These assumptions on $\Pi(t)$ gives $d \Pi(t)=r \Pi(t) d t$.

Let the option price value has the form

$$
G\left(t, s_{t}\right)=\int_{-T}^{0} e^{-r \theta} F(s(t+\theta), s(t), t) d \theta
$$

where $F \in C^{0,2,1}\left(\mathbb{R} \times \mathbb{R}^{2} \times \mathbb{R}^{+}\right)$.

\section{Lemma 3.1. (Itô Formula)}

Suppose $s(t)$ is given by (3.1) and a functional $G: \mathbb{R}^{+} \times C \rightarrow \mathbb{R}$ has the form

$$
G\left(t, s_{t}\right)=\int_{-\tau}^{0} g(\theta) F\left(s_{t}(\theta), s_{t}(0), t\right) d \theta
$$

where $F \in C^{0,2,1}\left(\mathbb{R} \times \mathbb{R} \times \mathbb{R}^{+}\right)$and $g \in C^{1}([-\tau, 0], \mathbb{R})$.

Hence in view of the classical Itô formula, we have

$$
\begin{aligned}
G\left(t, s_{t}\right)= & G(0, \zeta)+\int_{0}^{t} A G\left(s, S_{s}\right) d s+\int_{0}^{t} \sigma\left(s, S_{s}\right) S(s) B G\left(s, S_{s}\right) d w(s) \\
& +\int_{0}^{t} \int_{\mathbb{R}}\left\{F\left(t, s\left(t^{-}\right)+\gamma(s, z)\right)-F\left(t, s\left(t^{-}\right)\right)-F\left(t, S(t)^{-}\right)\right\} N(d t, d z)
\end{aligned}
$$

where for $(t, x) \in \mathbb{R}^{+} \times C$.

$$
\begin{aligned}
A G(t, y)= & g(0) F\left(x_{0}, x_{0}, t\right)-g(-\tau) F\left(x(-\tau), x_{0}, t\right)- \\
& \int_{-\tau}^{0} g^{\prime}(\theta) F(x(\theta), x 0, t) d \theta+\int_{-\tau}^{0} g(\theta) L F(x(\theta), x 0, t) d \theta \\
& +\int_{\mathbb{R}}\left\{F\left(t, x\left(t^{-}\right)+\gamma(x, z)\right)-F\left(t, x\left(t^{-}\right)\right)\right\} N(d t, d z),
\end{aligned}
$$


with

$$
\begin{aligned}
L F(x(\theta, x(0), t)= & r x(0) F_{2}^{\prime}(x(\theta, x(0), t)+ \\
& \frac{\sigma^{2}(t, x) x^{2}(0)}{2} F_{22}^{\prime \prime}\left(x(\theta, x(0), t)+F_{3}^{\prime}(x(\theta, x(0), t)\right. \\
& +\int_{\mathbb{R}}\left\{F(x+\gamma(x, z))-F(x)-F_{2}^{\prime} \cdot \gamma(x, z)\right\} \nu(d z),
\end{aligned}
$$

where $F_{i}^{\prime}, i=1,2,3$ represents the derivative of $F$ with respect to the $i^{t h}$ argument.

\section{Portfolio concepts for financial markets driven by Le'vy process:}

Theorem 3.1. The option price value given by (3.2) satisfies the equation

$$
\begin{array}{r}
0=\left.F\right|_{\theta=0}-\left.e^{r \theta} F\right|_{\theta=-\tau}+\int_{-\tau}^{0} e^{-r \theta}\left\{\left(F_{3}^{\prime}+r s(t) F_{2}^{\prime}+\frac{1}{2} \sigma^{2}(t, s(t)) s^{2}(t) F_{22}^{\prime \prime}\right) d \theta+\right. \\
\left.\left.\int_{\mathbb{R}}\{F(s+\gamma(s, z))-F(s))-F_{2}^{\prime} \gamma(s, z)\right\} \nu(d z)\right\} d \theta .
\end{array}
$$

Proof. We sketch the proof as in [12] as follows:

By considering a portfolio consists of -1 derivative and $B G\left(t, s_{t}\right)$ shares. Then if $\Pi(t)$ represents the portfolio value we have

$$
\Pi(t)=-G\left(t, s_{t}\right)+B G\left(t, s_{t}\right) s(t)=-d G+d(B G S)=d(B G) S+B G d S-d F .
$$

Hence

$$
d \Pi(t)=-d G+B G d S
$$

Since we assume $B G$ is held constant during the time-step dt yields $d(B G)$ equal zero.

Substituting for $d G$ and $d S$ from equation (3.4) and (3.1) we get

$$
d \Pi=-\bar{A} G d t-\sigma s B G d w+B G(r s d t+\sigma s d w)
$$

where

$$
\bar{A}=A-\int_{\mathbb{R}}^{t}\left\{F\left(t, s\left(t^{-}\right)+\gamma(s, z)\right)-F\left(t, s\left(t^{-}\right)\right)-F\left(t, S(t)^{-}\right)\right\} N(d t, d z) .
$$

By considering risk-free during the time $d t$ gives

$$
d \Pi=r \Pi d t
$$


By equating the last equations we get

$$
\bar{A} G\left(t, s_{t}\right)=r G\left(t, s_{t}\right)
$$

which gives an equation for $F(S(t+\theta), S(t), t)$ in the form

$$
\begin{array}{r}
0=\left.F\right|_{\theta=0}-\left.e^{r \theta} F\right|_{\theta=-\tau}+\int_{-\tau}^{0} e^{-r \theta}\left\{\left(F_{3}^{\prime}+r s(t) F_{2}^{\prime}+\frac{1}{2} \sigma^{2}(t, s(t)) s^{2}(t) F_{22}^{\prime \prime}\right) d \theta+\right. \\
\left.\left.\int_{\mathbb{R}}\{F(s+\gamma(s, z))-F(s))-F_{2}^{\prime} \gamma(s, z)\right\} \nu(d z)\right\} d \theta .
\end{array}
$$

\section{Price Formula for European Call Option:}

\section{Theorem 4.1.(Black-Scholes PDE Type)}

In view of (3.3) the option price value $G\left(T, S_{T}\right)$ will has the form

$$
G\left(T, S_{T}\right)=\max (S(T)-K, 0)
$$

For simplification we assume that $G\left(T, S_{t}\right)$ takes the form

$$
G\left(t, s_{t}\right)=g_{1}(s(t), t)+\int_{-\tau}^{0} e^{-r \theta} g_{2}(s(t+\theta), t) d \theta,
$$

where $g_{1}(s(t), t)$ is a classical Black-Scholes call option price with variance assumed equal to a long-run variance rate $\mathrm{V}$, then

$$
g_{1}(s(t), t)=s(t) N\left(d_{1}\right)-k e^{-r(T-t)} N\left(d_{2}\right)
$$

where

$$
\begin{array}{r}
N(x)=\frac{1}{\sqrt{2 \pi}} \int_{-\infty}^{x} e^{-\frac{x^{2}}{2}} d x \\
d_{1}=\ln \left(\frac{s(t)}{k}\right)+\left(r+\frac{V}{2}\right)(T-t) \\
d_{2}=d_{1}-\sqrt{V(T-t)} .
\end{array}
$$

Then $G\left(t, S_{t}\right)$ satisfies the following equation:

$$
\begin{aligned}
\frac{\partial G}{\partial t}+r S(t) \frac{\partial g_{1}}{\partial S}+ & \frac{1}{2}\left[\sigma^{2}\left(t, s_{t}\right)\right] s^{2}(t) \frac{\partial^{2} g_{1}}{\partial s^{2}}+ \\
& \left.\int_{\mathbb{R}}\{F(s+\gamma(s, z))-F(s))-F_{2}^{\prime} \gamma(s, z)\right\} \nu(d z)=r G .
\end{aligned}
$$


Proof. By substituting (4.1) into (3.5) we obtain the equation for $g_{2}$ as follows

$$
\begin{aligned}
g_{2}(S(t), t)-e^{r \tau} g_{2}(S(t-\tau), t)+ & \int_{\text {tau }}^{0} e^{-r \theta} \frac{\partial g_{2}}{\partial t} d \theta \\
& =\frac{1}{2}\left(V-\sigma^{2}(t, S(t))\right) S^{2}(t) \frac{\partial^{2} g_{1}}{\partial S^{2}}+ \\
& \left.\int_{\mathbb{R}}\{F(s+\gamma(s, z))-F(s))-F_{2}^{\prime} \gamma(s, z)\right\} \nu(d z) .
\end{aligned}
$$

Since $g_{1}$ satisfies the classical Black-Scholes PDE we have:

$$
\frac{\partial g_{1}}{\partial t}+r S(t) \frac{\partial g_{1}}{\partial S}+\frac{1}{2}[V] S^{2}(t) \frac{\partial^{2} g_{1}}{\partial s^{2}}=r g_{1}
$$

By combining the last equation we get the following equation $G\left(t, S_{t}\right)$ of the form :

$$
\begin{aligned}
\frac{\partial G}{\partial t}+r S(t) \frac{\partial g_{1}}{\partial S}+ & \frac{1}{2}\left[\sigma^{2}\left(t, s_{t}\right)\right] s^{2}(t) \frac{\partial^{2} g_{1}}{\partial s^{2}}+ \\
& \left.\int_{\mathbb{R}}\{F(s+\gamma(s, z))-F(s))-F_{2}^{\prime} \gamma(s, z)\right\} \nu(d z)=r G,
\end{aligned}
$$

which is an integro-differential equation.

Remark. For pricing American Put Option we give the following discussion :

Suppose the functional (3.3) has the form $S(t)-\int_{-\tau}^{0} S(t+\xi) d \xi$ then the pricing formula for the European Put Option is to minimize the following functional overall $F_{t}$ stoping time, $\tau<\infty$ a.s. for all $t \geq 0$ :

$$
F\left(\tau, S_{\tau}\right)=E\left[\left(K-\left(S(\tau)-\int_{-T}^{0} S(t+\xi) d \xi\right)\right)^{+}\right]
$$

where $K>0$.

The dynamics of the stock prices are driven by the following stochastic differential equation:

$$
\begin{aligned}
d S(t)= & \mu(S(t)-S(t-T)) d t+\alpha\left(S(t)-\mu \int_{-T}^{0} S(t+\xi)\right) d w(t)+ \\
& \beta \int_{\mathbb{R}}\left(S\left(t^{-}\right)-\mu \int_{-T}^{0} S\left(t^{-}+\xi\right) d \xi\right) z \tilde{N} d t d z
\end{aligned}
$$

with the initial conditions $S(0)=y_{0} ; S(\theta)=y_{1}(\theta), \theta \in[-T, 0)$, where $\mu, \alpha, \beta$ are constants and $y:=$ $\left(y_{0}, y_{1}(.) \in C\right.$.

The assumptions $y_{0}-\mu \int_{-T}^{0} y_{1}(\xi) d \xi>0$ and $\nu \equiv 0$ on $(-\infty, 0]$ are imposed to ensure that positivity of the solution and which it is exist and the allowance only for positive jumps. 
The problem of finding the optimal exercise time of

$$
F\left(\tau, S_{\tau}\right)=E\left[\left(K-\left(S(\tau)-\int_{-T}^{0} S(t+\xi) d \xi\right)\right)^{+}\right]
$$

is strictly connected to the corresponding optimal stoping problem of such stochastic system. This problem was studied by [13] where the solution is obtained by rewriting the problem in infinite dimension from which they found the condition under which the problem is reduced to one dimensional case which yields explicit solution.

Conflicts of Interest: The author(s) declare that there are no conflicts of interest regarding the publication of this paper.

\section{REFERENCES}

[1] A. Chojnowska-Michalik, Representation theorem for general stochastic delay equations, Bull. Acad. Polon. Sci. Ser. Sci. Math. Astronom. Phys. 26 (1978), 635-642.

[2] G. Da Prato, J. Zabczyk, Stochastic equations in infinite dimensions, Encyclopedia of Mathematics and its Applications, Cambridge University Press, Cambridge (UK), 1992.

[3] I. Elsanousi, B. Pksendal, A. Sulem, Some solvable stochastic control problems with delay, Stoch. Stoch. Rep. 71 (2000), 69-89.

[4] J.C. Hull, A. White, The pricing of options on assets with stochastic valatilities, J. Finance, 42 (1987), 281-300.

[5] S.-E.A. Mohammed, Stochastic Differential Systems With Memory: Theory, Examples and Applications, in: L. Decreusefond, B. Øksendal, J. Gjerde, A.S. Üstünel (Eds.), Stochastic Analysis and Related Topics VI, Birkhäuser Boston, Boston, MA, 1998: pp. 1-77.

[6] F. Black, M. Scholes, The pricing of options and corporate liabilities, J. Polit. Econ. 81 (1973), 637-654.

[7] B. Oksendal, A. Sulem, Applied stochastic control of jump diffusions, Springer, Berlin, 2005.

[8] M. Arriojas, Y. Hu, S.-E. Mohammed, G. Pap, A delayed black and scholes formula, Stoch. Anal. Appl. 25 (2007), 471-492.

[9] D. Applebanm, Le'vy process and stochastic calculus, Cambridge Studies in Advanced Mathematics Vol. 116, Cambridge University Press, Cambridge, 2009.

[10] P.E. Protter, Stochastic integration and differential equations, Springer, Berlin, 2005.

[11] M. Reiß, M. Riedle, O. van Gaans, Delay differential equations driven by Lévy processes: Stationarity and Feller properties, Stoch. Proc. Appl. 116 (2006), 1409-1432.

[12] Y. Kazmerchuk, A. Swishchuk, J. Wu, The pricing of options for securities markets with delayed response, Math. Computers Simul. 75 (2007), 69-79.

[13] S. Federico, B.K. Øksendal, Optimal Stopping of Stochastic Differential Equations with Delay Driven by Lévy Noise, Potential Anal. 34 (2011), 181-198. 